\title{
Correlation among SDS Sedimentation Value, Swelling Index of Glutenin and Solvent Retention Capacity of Spring Wheat
}

\author{
Deyong ZHAO*, Lei WANG, Yunting LEI \\ Northwest Institute of Plateau Biology, Chinese Academy of Sciences, Key Laboratory of Adaptation and Evolution of Plateau Biota, \\ 23 Xin'ning Road, Xi'ning, Qinghai Province, 810001, P.R. China; zhaodeyong07@mails.gucas.ac.cn (*corresponding author)
}

\begin{abstract}
The determination of flour end-use quality of progenies is a routine process in high-quality wheat breeding program. Traditional determination involves larger amount offlour and specific instruments, meanwhile, is expensive and time-consuming. A rapid and accurate method which could predict the quality of early generation wheat lines is in high demand in the wheat breeding program. Clarifying the relationships among different prediction indexes is the prerequisite for devising a new prediction method screening the high-quality progenies. This study aimed to clarify the correlations between SDS sedimentation value, SIG and SRCs for both wholemeal flour and flour, as well as to study which parameter could be applied to predict the flour quality of spring wheat rapidly and conveniently. The result of the study showed that WSRC correlated significantly with all the parameters determined, the four different SRCs varied significantly between hard wheat and soft wheat, while SDS sedimentation value and SIG showed no significant difference between hard wheat and soft wheat. SRC test may provide a convenient method for predicting the quality of early generation wheat lines in wheat breeding.
\end{abstract}

Keywords: Qinghai-Tibet plateau, SDS sedimentation value, solvent retention capacity, spring wheat, swelling index of glutenin

Abbreviations: LASRC-lactic acid SRC; SDS-sodium dodecylsulphate; SDSSV-sodium dodecylsulphate sedimentation value; SIGswelling index of glutenin; SODSRC-sodium carbonate SRC; SUCSRC-sucrose SRC; SRC-solvent retention capacity; WSRC-water SRC

\section{Introduction}

Spring wheat is one of the main crops in the QinghaiTibet plateau (China); it provides the staple food for local people. With the increasing need of food quality, improving the quality of spring wheat has always been an important aim in the breeding programme in this region. Quality determination of the progenies is a necessary step during the quality-improving programme of spring wheat. Determination of the wheat quality by traditional method is expensive and time-consuming, therefore, a rapid and convenient determination method of the progenies of hybridization which could facilitate the breeding programme is in high demand.

SDS sedimentation value is a practical index which associates tightly with protein content and protein composition, it was modified from zeleny sedimentation (Axford et al., 1979; Zeleny, 1947) test and firstly proposed in 1970s (McDermott and Redman 1977; ). It is generally accepted that the wheat with higher SDS sedimentation value tend to have higher baking-quality. The SDS-sedimentation test correlates better with loaf volume than the Zeleny sedimentation test (Axford et al., 1979).

SIG (swelling index of glutenin) correlates significantly with many quality parameters, including rheological property (Wang and Kovacs, 2002a, b). It is a rapid and conve- nient method to predict the quality of flour/wholemeal as it involves less flour/wholemeal.

SRC (solvent retention capacity), which was adopted by AACC as method 56-11, is used to measure the solvent-holding capacity of flour, it includes four kinds of index: lactic acid SRC (LASRC), sodium carbonate SRC (SODSRC), sucrose SRC (SUCSRC) and water SRC (WSRC). Generally, lactic acid SRC (LASRC) is associated with glutenin characteristics; sodium carbonate SRC (SODSRC) with starch damage; sucrose SRC (SUCSRC) with pentosan content and gliadin characteristics; the water SRC (WSRC) indicates the integrative property of all the four constituents (Gaines, 2000).

The spring wheat flour has been poor for many years in Qinghai-Tibet plateau, it is high time for the local breeders to improve the quality of spring wheat, and develop a rapid and accurate method to predict the quality of early generation materials. For some researchers and breeders, especially those who are in rural regions, to conduct largescale screening of the progenies, it is more convenient to measure the quality using wholemeal flour (mixture of flour and bran) than flour. However, can wholemeal flour be utilized instead of flour to predict the quality of progenies? What is the relationship among different quality parameters? Based on these practical considerations, the study was conducted and aimed to (1) clarify the correla- 
tion between SDS sedimentation value, SIG and SRCs for both wholemeal flour and flour, (2) to study which parameter could be applied to predict the flour quality of spring wheat rapidly and conveniently.

\section{Material and methods}

\section{Materials}

This experiment involved 55 spring wheat cultivars from Qinghai-Tibet Plateau, China (Fig. 1). These cultivars coverage almost released wheat cultivars in this region in the past 50 years. Twenty five cultivars are soft wheats while the others are hard wheat (Tab. 1).

\section{Determination of SDS sedimentation value}

SDS sedimentation value was determined according to Chinese standard method (GB/T15685-1995). 5.0 g flour or $6.0 \mathrm{~g}$ wholemeal flour was used for flour or wholemeal flour respectively.

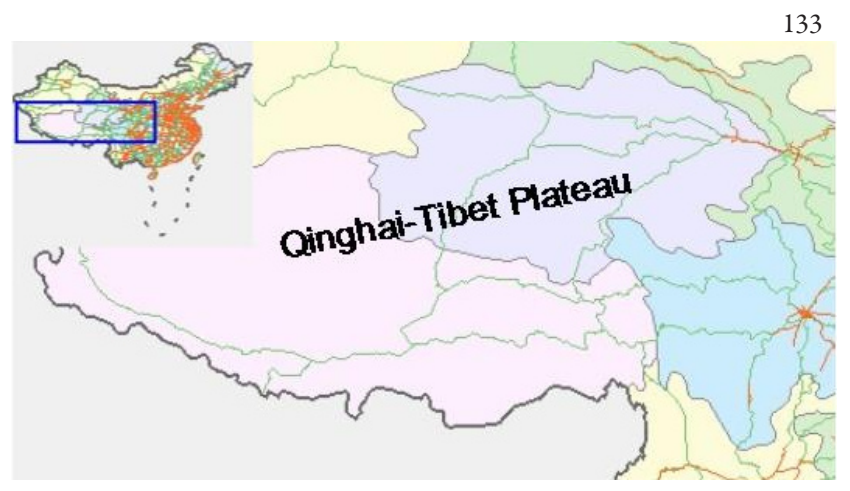

Fig. 1. Location of Qinghai-Tibet Plateau in the map of China

Determination of swelling index of glutenin

Determination of swelling index of glutenin was modified from the method of Wang and Kovacs (2002a). One hundred fifty $\mathrm{mg}$ flour/wholemeal flour was added into the centrifuge tube. The flour was scattered by adding $3 \mathrm{ml}$ distilled water, then the centrifuge tube was kept in water (constant temperature $24^{\circ} \mathrm{C}$ ) for $20 \mathrm{~min}$, two times oscil-

Tab. 1. Name list of the wheat cultivars in this study

\begin{tabular}{|c|c|c|c|c|c|}
\hline No & Cultivars & Hard or soft & No & Cultivars & Hard or soft \\
\hline 1 & 'Abbondanza' & Soft & 29 & 'Huzhuhong' & Soft \\
\hline 2 & 'Caoxuan No5’ & Hard & 30 & 'Lantian No3' & Hard \\
\hline 3 & 'Chaichun 018' & Hard & 31 & 'Lemai No5' & Soft \\
\hline 4 & 'Chaichun 044' & Hard & 32 & 'Lemai No6' & Soft \\
\hline 5 & 'Chaichun 236 ' & Soft & 33 & 'Minhe 588' & Soft \\
\hline 6 & 'Chaichun 901' & Hard & 34 & 'Minhe 665' & Hard \\
\hline 7 & 'Dongchun Nol' & Soft & 35 & 'Minhe 853' & Soft \\
\hline 8 & 'Ganchun 20’ & Hard & 36 & 'Mobo' & Hard \\
\hline 9 & 'Gaoyuan 115’ & Hard & 37 & 'Moyin Nol' & Soft \\
\hline 10 & 'Gaoyuan 158' & Soft & 38 & 'Moyin No2' & Hard \\
\hline 11 & 'Gaoyuan 175’ & Soft & 39 & 'Ningchun 26 ' & Hard \\
\hline 12 & 'Gaoyuan 182' & Soft & 40 & 'Qingchun 144' & Hard \\
\hline 13 & 'Gaoyuan 205' & Hard & 41 & 'Qingchun 254' & Hard \\
\hline 14 & 'Gaoyuan 314' & Soft & 42 & 'Qingchun 37’ & Hard \\
\hline 15 & 'Gaoyuan 356 & Hard & 43 & 'Qingchun 415' & Hard \\
\hline 16 & 'Gaoyuan 363' & Hard & 44 & 'Qingchun 533' & Soft \\
\hline 17 & 'Gaoyuan 448' & Hard & 45 & 'Qingchun 570' & Soft \\
\hline 18 & 'Gaoyuan 465' & Soft & 46 & 'Qingchun 587’ & Hard \\
\hline 19 & 'Gaoyuan 466 & Soft & 47 & 'Qingchun 891' & Hard \\
\hline 20 & 'Gaoyuan 506 & Hard & 48 & 'Qingchun 952' & Soft \\
\hline 21 & 'Gaoyuan 584' & Hard & 49 & 'Qingnong 469' & Hard \\
\hline 22 & 'Gaoyuan 602' & Hard & 50 & 'Qingnong 524' & Soft \\
\hline 23 & 'Gaoyuan 913’ & Soft & 51 & 'Shanhan 901' & Hard \\
\hline 24 & 'Gaoyuan $\mathrm{V}_{0} 28$ ' & Hard & 52 & 'Tongmai Nol' & Soft \\
\hline 25 & 'Hanhai $304 '$ & Hard & 53 & 'Xiangnong No3' & Hard \\
\hline 26 & 'Humai 11' & Soft & 54 & 'Xinzhe No9' & Soft \\
\hline 27 & 'Humai 12' & Soft & 55 & 'Zhangchun 811' & Soft \\
\hline 28 & 'Humai 13' & Hard & & & \\
\hline
\end{tabular}


134

lation was needed within this interval. Three $\mathrm{ml}$ SDS-lactic acid solution was added into centrifuge tube, and kept in $24^{\circ} \mathrm{C}$ water for $20 \mathrm{~min}$ after oscillation. Centrifuged (centrifugal force, $300 \times \mathrm{g}$ ) for $5 \mathrm{~min}$, then the supernatant was removed. The SIG value was calculated by the equation: SIG=weight of wet panada/weight of flour. For the wholemeal flour, the SIG= weight of wet panada/weight of wholemeal flour.

\section{Determination of solvent retention capacity}

The SRC tests of flour and wholemeal flour were conducted according to AACC method 56-11 (AACC, 2000 ) with modification. One gram flour or wholemeal flour were added into $15 \mathrm{ml}$ centrifuge tube with a conical bottom. Then, $5.0 \mathrm{ml}$ distilled water and the corresponding solutions, $5.0 \%(\mathrm{w} / \mathrm{w})$ sodium carbonate in water, $50.0 \%(\mathrm{w} / \mathrm{w})$ sucrose in water, $5.0 \%(\mathrm{w} / \mathrm{w})$ lactic acid in water, were added and the mixtures were oscillated for 5 $s$ to scatter the flour. Samples were centrifuged $(15 \mathrm{~min}$, $1000 \mathrm{~g}$, room temperature) after being kept at room temperature for $20 \mathrm{~min}$. Supernatant was removed from the tubes, the pellet was weighed after drained for $15 \mathrm{~min}$. The SRC values were calculated as follows:

SRC $(\%)=[$ Wet pellet $/$ flour $\times 86 /(100$-flour moisture) -1$] \times 100 \%$

\section{Statistical analysis}

The T-test and correlation analysis of experimental statistics was carried out using the software SPSS 16.0.

\section{Result and discussion}

T-test was conducted to study the difference of each determined parameter between hard wheat and soft wheat
(Tab. 2 and 3). For both flour and wholemeal flour, the WSRC, SUCSRC, SODSRC, LASRC varied significantly between hard wheat and soft wheat. The difference between hard wheat WSRC and soft wheat WSRC was significant at 0.01 level, while that of SUCSRC, SODSRC, LASRC was significant at 0.05 level. By contrast, the SIG and SDSSV could not distinguish the hard wheat from soft wheat. The value of each determined parameter using flour was a little larger than the corresponding value using wholemeal, such a result indicated that the function of bran was different from that of flour. Besides, it can be deduced that the bran had lower capacity to swell or absorb the solvent.

Tab. 4 and Tab. 5 showed the correlation coefficient among all quality parameters determined for both wholemeal flour and flour respectively. The correlation coefficient for flour was a little larger than that of wholemeal flour, it provided further evidence that the function of bran was different from that of flour. However, using the wholemeal for the determination of quality parameter could also get a nice result. For example, the WSRC correlated significantly with SUCSRC, SODSRC, LASRC at 0.01 level, SUCSRC correlated significantly with SODSRC, LAS$\mathrm{RC}$ at 0.01 level, SIG correlated significantly with SDSSV at 0.01 level, and WSRC correlated significantly with SIG, SDSSV at 0.05 level, SUCSRC correlated significantly with SIG at 0.05 level, SODSRC correlated significantly with LASRC at 0.05 level. The correlation among different quality parameters was less affected by using wholemeal flour than using flour.

For a long time, in the Qinghai-tibet plateau arable region, the wheat breeding aim has been focused on the high-yield rather than the high quality of flour, partly due to the limitation of technology and instrument in quality

Tab. 2. Flour quality parameters of the soft and hard wheat

\begin{tabular}{ccccccc}
\hline & WSRC & SUCSRC & SODSRC & LASRC & SIG & SDSSV $(\mathrm{ml})$ \\
\hline Hard wheat & $95.46^{* *}$ & $138.97^{*}$ & $105.67^{*}$ & $102.95^{*}$ & 3.94 & 30.73 \\
Soft wheat & $110.35^{* *}$ & $130.45^{*}$ & $98.86^{*}$ & $98.22^{*}$ & 3.99 & 28.85 \\
\hline
\end{tabular}

“*** and "**" indicate the differences are significant at 0.01 and 0.05 probability level, respectively

Tab. 3. wholemeal flour quality parameters of the soft and hard wheat

\begin{tabular}{ccccccc}
\hline & WSRC & SUCSRC & SODSRC & LASRC & SIG & SDSSV $(\mathrm{ml})$ \\
\hline Hard wheat & $93.13^{* *}$ & $137.20^{*}$ & $102.00^{*}$ & $101.83^{*}$ & 3.83 & 30.26 \\
Soft wheat & $109.95^{* *}$ & $129.25^{*}$ & $96.74^{*}$ & $97.12^{*}$ & 3.87 & 28.82 \\
\hline
\end{tabular}

“*** and "**" indicate the differences are significant at 0.01 and 0.05 level respectively

Tab. 4. Correlation among SIG, SDS, SRCs of wholemeal flour

\begin{tabular}{cccccc}
\hline & WSRC & SUCSRC & SODSRC & LASRC & SIG \\
\hline SUCSRC & $0.7242^{* *}$ & & & & \\
SODSRC & $0.8013^{* *}$ & $0.7785^{* *}$ & & & \\
LASRC & $0.6276^{* *}$ & $0.5897^{* *}$ & $0.4786^{*}$ & & \\
SIG & $0.4653^{*}$ & $0.3986^{*}$ & 0.2032 & 0.2574 & \\
SDSSV & $0.3576^{*}$ & 0.0046 & 0.2010 & 0.2270 & $0.5686^{* *}$ \\
\hline
\end{tabular}

"*** and "**" indicate the correlations are significant at 0.01 and 0.05 level respectively
Tab. 5. Correlation among SIG, SDS, SRCs of flour

\begin{tabular}{cccccc}
\hline & WSRC & SUCSRC & SODSRC & LASRC & SIG \\
\hline SUCSRC & $0.8564^{* *}$ & & & & \\
SODSRC & $0.8986^{* *}$ & $0.8103^{* *}$ & & & \\
LASRC & $0.7540^{* *}$ & $0.6120^{* *}$ & $0.4866^{*}$ & & \\
SIG & $0.5762^{* *}$ & $0.4216^{*}$ & 0.2203 & 0.2658 & \\
SDSSV & $0.4078^{*}$ & 0.0242 & 0.2108 & 0.2306 & $0.6018^{* *}$ \\
\hline
\end{tabular}

"**” and "** indicate the correlations are significant at 0.01 and 0.05 level respectively 
determination. Empirically speaking, successful screen of the elite progenies involves a large number of progeny materials to be determined. Consequently, a rapid and convenient measurement of wheat quality would facilitate the breeding programme in the long run.

The SIG test needs the least flour $(150 \mathrm{mg})$ among the SDSSV, SIG and SRC. It correlated with SODSRC, LASRC not significantly. SDSSV involved 5.0 g flour or 6.0 $\mathrm{g}$ wholemeal flour in the test. No significant correlations were determined between SDSSV and other parameters, except the correlation between SDSSV and WSRC, SIG. This might result from the genotype difference of wheats determined. In this study, $1.0 \mathrm{~g}$ flour or wholemeal flour was used in SRC test. The correlation among different SRCs were significant, as well as the correlation between WSRC and SIG, SDSSV, the correlation between SUCSRC and SIG.

Previous studies indicated that SODSRC was positively correlated with WSRC, SUCSRC, while no correlation was detected between SODSRC with LASRC (Gaines, 2000; Guttieri et al., 2004; Pasha et al., 2009). However, in the present study, SODSRC show significantly positive correlations with all the other SRCs, i.e. WSRC, SUCSRC and LASRC. Former researchers found that SUCSRC was negatively correlated with SDS sedimentation volume (Guttieri et al., 2001; Pasha et al., 2009), in this study, SUCSRC was subtle positively correlated with SDSSV. Such differences might partially result from the different materials were applied in different researches. However, the highest correlation was detected between WSRC and SODSRC, which is in accordance to previous study (Pasha et al., 2009; Ram et al., 2005).

Wheat can be classified into hard or soft type due to the grain texture. To our knowledge, none of the previous studies regarding SRCs, SIG of wheat flour was conducted to study the difference between hard and soft wheat (Gaines, 2000; Guttieri et al., 2001; Guttieri et al., 2004; Pasha et al., 2009; Ram et al., 2005; Slade and Levine, 1994; Wang and Kovacs, 2002a; Wang and Kovacs, 2002b; Zhang et al., 2007). In the present study, WSRC of soft wheat, for both flour and wholemeal flour, are larger than that of hard wheat; while SUCSRC, SODSRC and LASRC of soft wheat, for both flour and wholemeal flour, are smaller than that of hard wheat. Such differences suggest a promising way to develop determination method that can distinguish the soft and hard wheat. For SIG and SDSSV, no difference was detected between hard and soft wheat.

\section{Conclusions}

The SRC test was firstly developed by Slade and Levine (1994), and it was subsequently adopted as AACC method 56-11 in the year 2000; many wheat breeders applied SRC to predict the quality of early generation. In this study, for both wholemeal flour test and flour test, WSRC correlated significantly with SUCSRC, SODSRC, LAS-
RC, SIG and SDSSV at 0.05 or 0.01 level. On the other hand, the correlations among different SRCs were also significant using both wholemeal flour and flour. Besides, the SRC could also distinguish hard wheat from soft wheat. It can be deduced the measurement of SRC may provide a promising convenient method for predicting the quality of early generation wheat lines.

\section{References}

AACC (2000). Approved methods of the American Association of Cereal Chemists, $\left(10^{\text {th }} \mathrm{ed}\right.$.). The American Association of Cereal Chemists, St. Paul, MN.

Axford DWE, McDermott EE, Redman DG (1979). Note on the sodium dodecyl sulfate test of breadmaking quality: comparison with pelshenke and zeleny tests. Cereal Chem 56:582-584.

Chinese standard method for determination of wheat SDS sedimentation value (1995). GB/T15685-1995.

Gaines CS (2000). Collaborative study of methods for solvent retention capacity profiles (AACC Method 56-11). Cereal Foods World 45:303-306.

Guttieri MJ, Becker C, Souza EJ (2004). Application of wheat meal solvent retention capacity tests within soft wheat breeding population. Cereal Chem 81:261-266.

Guttieri MJ, Bowen D, Gannon D, O’Brien K, Souza E (2001). Solvent retention capacities of irrigated soft white spring flours. Crop Sci 41:1054-1061.

McDermott EE, Redman DG (1977). Small-scale tests of breadmaking quality. Flour Mill Bak Res Assoc Bull 6:200-213.

Pasha I, Anjum FM, Butt MS (2009). Genotypic variation of spring wheats for solvent retention capacities in relation to end-use quality. LWT-Food Sci Technol 42:418-423.

Wang C, Kovacs MIP (2002a). Swelling index of glutenin test. I. Method and comparison with sedimentation, gel-protein, and insoluble glutenin tests. Cereal Chem 79:183-189.

Wang C, Kovacs MIP (2002b). Swelling index of glutenin test. II. Application in predication of dough properties and enduse quality. Cereal Chem 79:190-196.

Ram S, Dawar V, Singh RP, Shoran J (2005). Application of solvent retention capacity tests for the prediction of mixing properties of wheat flour. J Cereal Sci 42:261-266.

Slade L, Levine H (1994). The Science of Cookies and Cracker Production, 23-141 p. In: Faridi H (Ed.). Structure-function relationships of cookies and cracker ingredients. Chapman and Hall, New York.

Zeleny L (1947). A simple sedimentation test for estimating the bread baking and gluten qualities of wheat flour. Cereal Chem 24:465-475.

Zhang Q, Zhang Y, Zhang Y, He Z, Peña RJ. (2007). Effects of solvent retention capacities, pentosan content, and dough rheological properties on sugar snap cookie quality in Chinese soft wheat genotypes. Crop Sci 47:656-664. 